05,11

\title{
Негейзенберговский ферримагентик с одноионной анизотропией
}

\author{
() О.А. Космачев, Е.А. Ярыгина, Я.Ю. Матюнина, Ю.А. Фридман \\ Крымский фредеральный университет им. В.И. Вернадского, \\ Симферополь, Республика Крым, Россия \\ E-mail: yuriifridman@gmail.com \\ Поступила в Редакцию 8 ноября 2021 г. \\ В окончательной редакции 12 ноября 2021 г. \\ Принята к публикации 18 ноября 2021 г.
}

\begin{abstract}
Исследовано влияние одноионной анизотропии типа „легкая плоскость“ на фазовые состояния ферримагнетика с подрешетками $S=1$ и $\sigma=1 / 2$ и негейзенберговским (билинейным и биквадратичным по спинам) обменным взаимодействием для подрешетки с $S=1$. Показано, что при различных соотношениях материальных параметров системы возможна реализация фазы с векторными параметрами порядка (ферримагнитная фаза) и фазы, характеризуемой как векторным, так и тензорными параметрами порядка (квадрупольноферримагнитная). Показано, что учет одноионной анизотропии меняет тип фазового перехода по сравнению с изотропным негейзенберговским ферримагнетиком. Построена фазовая диаграмма, а также определено условие компенсации спинов подрешеток.
\end{abstract}

Ключевые слова: ферримагнетик, биквадратичное обменное взаимодействие, одноионная анизотропия „легкая плоскость“, квадрупольно-ферримагнитная фаза, фазовый переход.

DOI: 10.21883/FTT.2022.03.52094.235

\section{1. Введение}

В последние годы возникла новая и активно развивающаяся область физики магнетизма - спинтроника, в которой активно исследуются и применяются скомпенсированные магнетики (см. [1-3]). Этот интерес связан с тем, что для них динамические параметры, такие как, частоты магнитного резонанса, скорости доменных стенок и ряд других, являются обменно усиленными. В работе [4] было показано, что спиновый ток активно влияет на магнитоупорядоченные системы с нулевым интегральным магнитным моментом, что позволяет использовать антиферромагнетики в спинтронике. Это обстоятельство, в принципе, позволяет повысить скорость работы систем записи и считывания информации [5-8], и существенно (до величин порядка $\mathrm{THz}$ ) повысить рабочую частоту генераторов с накачкой спиновым током [9-11]. Однако, несмотря на всю привлекательность антиферромагнетиков, они обладают высокой чувствительностью магнитного порядка к наличию дефектов, нарушающих подрешеточную структуру кристаллического образца, что затрудняет их применение в наносистемах. С другой стороны, для ферримагнетиков типа $\mathrm{GdFeCo}$, аморфных сплавов редкоземельных элементов с элементами группы железа, можно использовать стандартные нанотехнологии, такие же, как для классических материалов наномагнетизма, железа, никеля или пермаллоя. Хорошо известно, что эффекты обменного усиления динамических параметров, аналогичные тем, что известны для антиферромагнетиков, имеют место для ферримагнетиков, находящихся в окрестности точки компенсации подрешеток [12]. Таким образом, возникает возможность использовать фер- римагнетики, находящиеся вблизи точки компенсации, для различных устройств сверхбыстрой спинтроники. В недавних работах экспериментально и теоретически исследована сверхбыстрая (со скоростями порядка $\mathrm{km} / \mathrm{s}$ ) динамика доменных стенок $[13,14]$ и высокочастотная динамика ферримагнитных вихрей $[15,16]$. Предложена схема магнитного наногенератора на основе ферримагнетиков с накачкой спиновым током, работающего в диапазоне $\mathrm{THz}$ [17]. Эти обстоятельства делают детальное исследование различных аспектов спиновой динамики ферримагнетиков практически важными и актуальными (см. недавний обзор [18]). Кроме того, для ферримагнетиков (а именно, сплава редкоземельных и переходных металлов $\mathrm{GdFeCo}$ ) был обнаружен сверхбыстрый (за время порядка нескольких ps) переворот намагниченностей подрешеток под действием лазерного импульса с длительностью меньше $100 \mathrm{fs}[19,20]$. Оказалось, что этот эффект напрямую связан с наличием двух подрешеток, и в формировании эффекта существенную роль играет обусловленное обменным взаимодействием изменение модулей магнитных моментов подрешеток, такое, что их сумма остается постоянной $[21,22]$. Таким образом, для описания эффекта существенна чисто продольная эволюция магнитных моментов подрешеток.

Необходимо отметить что ряд вопросов физики ферримагнетиков изучен сравнительно слабо. В частности, отмеченный выше эффект переориентации наблюдался для ферримагнетика, содержащего как слабоанизотропные ионы, так и редкоземельные ионы, обладающие немалой одноионной анизотропией. Наличие немалой одноионной анизотропии приводит к существенно квантовым эффектам, не описываемым стандартной фено- 
менологической теорией [23]. Полное описание подобных эффектов требует учета динамики тензорных переменных, представляющих собой квантовые средние от операторов, билинейных по компонентам спина, что выходит за рамки уравнения Ландау-Лифшица $[24,25]$. Эффект квантового сокращения спина характерен для магнетиков с одноионной анизотропией типа „легкая плоскость“ [26-30]. Можно предположить, что эффект сокращения спина может быть предложен для описания сверхбыстрого продольного „переключения“ спинов [29-30].

Необходимо подчеркнуть, что эффект квантового сокращения спина реализуется не только в сильно анизотропных магнетиках, но и в так называемых негейзенберговских магнетиках. Под термином „негейзенберговские магнетики“ мы подразумеваем магнитоупорядоченные системы, в которых высшие спиновые инварианты вида $\left(\mathbf{S}_{1}, \mathbf{S}_{2}\right)^{n}$ со значениями $n$ до $2 S$, где $S-$ величина спина магнитного иона, играют важную роль как в формировании статических, так и динамических свойств [31-34]. Так, гамильтониан для изотропного обменного взаимодействия магнетика с $S=1$ содержит как билинейное $\left(\mathbf{S}_{1}, \mathbf{S}_{2}\right)$, так и биквадоатичное слагаемое $\left(\mathbf{S}_{1}, \mathbf{S}_{2}\right)^{2}[25,31,32-37]$. В недавних работах по исследованию динамических и статических свойств изотропного и обменно-анизотропного негейзенберговских ферримагнетиков с подрешетками $S=1$ и $\sigma=1 / 2$ и учетом биквадратичнго обменного взаимодействия в подрешетке со спином 1 [38,39], было показано, что в таких системах, в зависимости от соотношения материальных параметров, возможна реализация как ферримагнитной фазы, характеризуемой дипольными параметрами порядка, так и фазы, состояние которой описывается как дипольными, так и тензорными параметрами порядка (квадрупольно-ферримагнитной). Причем в этом „смешанном“ состоянии возможна компенсация магнитных моментов подрешеток, т. е. существует линия компенсации.

Таким образом, вопрос о свойствах негейзенберговских ферримагнетоков с учетом влияния одноионной анизотропии типа „легкая плоскость“ представляет не только академический интерес, но и важное прикладное значение.

\section{2. Модель}

В качестве исследуемой системы рассмотрим двухподрешеточный анизотропный магнетик со спином магнитного иона первой подрешетки $S=1$ и второй $\sigma=1 / 2$, и негейзенберговским обменным взаимодействием для подрешетки с $S=1$. При этом в первой подрешетки учитывается, как билинейное обменное, так и биквадратичное обменные взаимодействия, а также одноионная анизотропия типа „легкая плоскость“. Гамильтониан такой системы можно представить в виде

$$
\begin{aligned}
H= & -\frac{1}{2} \sum_{n, n^{\prime}}\left[J^{(2)}\left(n-n^{\prime}\right)\left(\mathbf{S}_{n}, \mathbf{S}_{n^{\prime}}\right)+K\left(n-n^{\prime}\right)\left(\mathbf{S}_{n}, \mathbf{S}_{n^{\prime}}\right)^{2}\right] \\
& -\frac{1}{2} \sum_{m, m^{\prime}} J^{(1)}\left(m-m^{\prime}\right)\left(\boldsymbol{\sigma}_{m} \boldsymbol{\sigma}_{m^{\prime}}\right) \\
& -\frac{1}{2} \sum_{n, m} A(n-m)\left(\boldsymbol{\sigma}_{m} \mathbf{S}_{n}\right)+\frac{\beta}{2} \sum_{n}\left(S_{n}^{x}\right)^{2}
\end{aligned}
$$

где $J^{(1)}>0-$ константа обменного взаимодействия для подрешетки со спином $\sigma=1 / 2 ; J^{(2)}>0, K>0-$ константы билиненйного и биквадратичного обменных взаимодействий для $S=1 ; A<0-$ константа межподрешеточного взаимодействия, $\beta>0-$ константа одноионной анизотропии типа „легкая плоскость“ (базисная плоскость ZOY). Дальнейшее рассмотрение будем проводить для случая низких температур $\left(T \ll T_{\mathrm{N}}, T_{\mathrm{N}}-\right.$ температура Нееля).

Изменение фазовых состояний связано с изменением величины материальных параметров (и их соотношения между собой) [24,32,36,38-41]. Вариация параметров системы может происходить, например, путем изменения концентрации магнитных ионов, или приложением внешних механических напряжений, приводящих к деформации кристаллической решетки. В контексте данной работы не принципиально, каким образом происходит изменение материальных констант в рассматриваемой модели.

В качестве оси квантования выберем ось $O Z$. Тогда среднее значение спина для первой подрешетки будет параллельно этой оси, а второй подрешетки - антипараллельно этой оси. Такая ориентация магнитных моментов подрешеток связана с тем, что константа межподрешеточного обменного взаимодействия $A<0$, что определяет антипараллельную ориентацию магнитных моментов подрешеток. Для удобства вычислений подвернем вторую подрешетку так, чтобы направления осей квантования обеих подрешеток совпадали. Унитарный поворот $U(\varphi)=\prod_{l} \exp \left(i \varphi \sigma_{l}^{x}\right)$ на угол $\varphi=\pi$ приводит к следующим преобразованиям компонент оператора спина второй подрешетки

$$
\sigma_{m}^{x} \rightarrow \sigma_{m}^{x}, \quad \sigma_{m}^{y} \rightarrow-\sigma_{m}^{y}, \quad \sigma_{m}^{z} \rightarrow-\sigma_{m}^{z} .
$$

Необходимо отметить, что при таких преобразованиях сохраняются стандартные коммутационные соотношения для компонент спиновых операторов.

Дальнейшие вычисления будем проводить, используя операторы Стивенса [42], поскольку средние значения этих операторов реализуют полный набор динамических переменных системы. 
Тогда гамильтониан исследуемой системы принимает вид

$$
\begin{aligned}
& H=-\frac{1}{2} \sum_{m, m^{\prime}} J^{(1)}\left(m-m^{\prime}\right)\left(\sigma_{m}^{x} \sigma_{m^{\prime}}^{x}+\sigma_{m}^{y} \sigma_{m^{\prime}}^{y}+\sigma_{m}^{z} \sigma_{m^{\prime}}^{z}\right) \\
& -\frac{1}{2} \sum_{n, n^{\prime}}\left[J^{(2)}\left(n-n^{\prime}\right)-\frac{K\left(n-n^{\prime}\right)}{2}\right]\left(S_{n}^{x} S_{n^{\prime}}^{x}+S_{n}^{y} S_{n^{\prime}}^{y}+S_{n}^{z} S_{n^{\prime}}^{z}\right) \\
& -\frac{1}{4} \sum_{n, n^{\prime}} K\left(n-n^{\prime}\right)\left(\frac{1}{3} O_{2 n}^{0} O_{2 n^{\prime}}^{0}+O_{2 n}^{1} O_{2 n^{\prime}}^{1}+\tilde{O}_{2 n}^{1} \tilde{O}_{2 n^{\prime}}^{1}\right. \\
& \left.+O_{2 n}^{2} O_{2 n^{\prime}}^{2}+\tilde{O}_{2 n}^{2} \tilde{O}_{2 n^{\prime}}^{2}\right)-\frac{1}{2} \sum_{m, n} A(m-n)\left(\sigma_{m}^{x} S_{n}^{x}-\sigma_{m}^{y} S_{n}^{y}\right. \\
& \left.-\sigma_{m}^{z} S_{n}^{z}\right)+\frac{\beta}{12} \sum_{n} O_{2 n}^{2}+\frac{\beta}{8} \sum_{n}\left(S_{n}^{+} S_{n}^{-}+S_{n}^{-} S_{n}^{+}\right)
\end{aligned}
$$

где $\quad S^{ \pm}=S^{x} \pm i S^{y} ; \quad O_{2}^{0}=3\left(S^{z}\right)^{2}-S(S+1) ; \quad O_{2}^{1}=$ $=\frac{1}{2}\left[S^{z},\left(S^{+}+S^{-}\right)\right]_{+} ; \tilde{O}_{2}^{1}=\frac{1}{2 i}\left[S^{z},\left(S^{+}-S^{-}\right)\right]_{+} \tilde{O}_{2}^{2}=\frac{1}{2 i}$ $\times\left[\left(S^{+}\right)^{2}-\left(S^{-}\right)^{2}\right]-$ операторы Снивенса.

Выделяя в гамильтониане (2) средние поля, связанные как с дипольными параметрами порядка $\left\langle S^{z}\right\rangle$, так и с квадрупольными $\left(q_{2}^{t}=\left\langle O_{2}^{t}\right\rangle\right)$, получим одноузельный гамильтониан

$$
\begin{aligned}
H_{0}= & \bar{H}_{\sigma} \sigma_{n}^{z}+\bar{H}_{S} S_{n}^{z}-B_{2}^{0} O_{2 n}^{0}-B_{2}^{2} O_{2 n}^{2} \\
& +\frac{\beta}{8}\left(S^{+} S^{-}+S^{-} S^{+}\right)+\Delta
\end{aligned}
$$

где

$$
\begin{gathered}
\bar{H}_{S}=\left(J_{0}^{(2)}-K_{0} / 2\right)\left\langle S^{z}\right\rangle-\frac{1}{2} A_{0}\left\langle\sigma^{z}\right\rangle, \\
\bar{H}_{\sigma}=J_{0}^{(1)}\left\langle\sigma^{z}\right\rangle-\frac{1}{2} A_{0}\left\langle S^{z}\right\rangle, \\
B_{2}^{0}=\frac{K_{0}}{6} q_{2}^{0}, \quad B_{2}^{2}=\frac{K_{0}}{2} q_{2}^{2}-\frac{\beta}{12}, \\
\Delta=\frac{1}{2} J_{0}^{(1)}\left\langle\sigma^{z}\right\rangle^{2}+\frac{1}{2}\left(J_{0}^{(2)}-\frac{K_{0}}{2}\right)\left\langle S^{z}\right\rangle^{2} \\
+\frac{K_{0}}{4}\left(\frac{\left(q_{2}^{0}\right)^{2}}{3}+\left(q_{2}^{2}\right)^{2}\right)-\frac{1}{2} A_{0}\left\langle S^{z}\right\rangle\left\langle\sigma^{z}\right\rangle .
\end{gathered}
$$

Здесь $J_{0}^{(1)}, J_{0}^{(2)}, K_{0}$ и $A_{0}$ - нулевые фурье-компоненты соответствующих обменных интегралов.

Корректный учет как одноионной анизотропии, так и биквадратичного обменных взаимодействий удается провести в рамках диаграммной техники для операторов Хаббарда [43-46]. Эти операторы строятся на базисах собственных функций операторов $S^{z}(|M\rangle), M=-1,0,1$ и $\sigma^{z}(|m\rangle), \quad m=-1 / 2,1 / 2$ для первой $X^{M^{\prime} M}=\left|M^{\prime}\right\rangle\langle M|$ и второй $Y^{m^{\prime} m}=\left|m^{\prime}\right\rangle\langle m|$ подрешеток, соответственно, и описывают переход магнитного иона из состояния $M^{\prime}$ в состояние $M$ и из состояния $m^{\prime}$ в состояние $m$. Связь спиновых операторов и операторов Стивенса с операторами Хаббарда имеет вид

$$
S^{z}=X^{11}-X^{-1-1}, \quad O_{2}^{2}=X^{1-1}+X^{-11},
$$

$$
\begin{gathered}
O_{2}^{0}=X^{11}-2 X^{00}+X^{-1-1}, \\
\sigma^{z}=\frac{1}{2}\left(Y^{\frac{1}{2} \frac{1}{2}}-Y^{-\frac{1}{2}-\frac{1}{2}}\right), \quad \sigma^{+}=Y^{\frac{1}{2}-\frac{1}{2}}, \quad \sigma^{-}=\left(\sigma^{+}\right)^{+} .
\end{gathered}
$$

Тогда в терминах операторов Хаббарда одноузельный гамильтониан (3) можно представить в виде

$$
\begin{aligned}
H_{0}= & -\frac{1}{2} \bar{H}_{\sigma}\left(Y^{\frac{1}{2} \frac{1}{2}}-Y^{-\frac{1}{2}-\frac{1}{2}}\right)-\bar{H}_{S}\left(X^{11}-X^{-1-1}\right) \\
& -B_{2}^{2}\left(X^{1-1}+X^{-11}\right)-B_{2}^{0}\left(X^{11}-2 X^{00}+X^{-1-1}\right) \\
& +\frac{\beta}{4}\left(X^{11}+2 X^{00}+X^{-1-1}\right)+\Delta
\end{aligned}
$$

Как видно, гамильтониан (5) является недиагональным, и для его диагонализации используем унитарное преобразование [44]:

$$
\tilde{H}_{0}=U(\alpha) H_{0} U^{+}(\alpha)
$$

явный вид которого: $U(\alpha)=1+(\cos \alpha-1)\left(X^{11}+X^{-1-1}\right)$ $+\sin \alpha\left(X^{1-1}-X^{-11}\right)$.

В результате чего получим гамильтониан (5) в диагональном виде

$$
\tilde{H}_{0}=E_{1} X^{11}+E_{0} X^{00}+E_{-1} X^{-1-1}+\varepsilon_{\frac{1}{2}} Y^{\frac{1}{2} \frac{1}{2}}+\varepsilon_{-\frac{1}{2}} Y^{-\frac{1}{2}-\frac{1}{2}},
$$

где

$$
\begin{gathered}
E_{1}=-B_{2}^{0}-\frac{\beta}{4}-\bar{H}_{S} \cos 2 \alpha-B_{2}^{2} \sin 2 \alpha+\Delta, \\
E_{0}=-2 B_{2}^{0}+\frac{\beta}{2}+\Delta, \\
E_{-1}=-B_{2}^{0}-\frac{\beta}{4}+\bar{H}_{S} \cos 2 \alpha+B_{2}^{2} \sin 2 \alpha+\Delta, \\
\varepsilon_{\frac{1}{2},-\frac{1}{2}}=\mp \bar{H}_{\sigma}\left\langle\sigma^{z}\right\rangle,
\end{gathered}
$$

энергетические уровни магнитных ионов первой и второй подрешеток, соответственно.

Волновые функции подрешеток имеют вид

$$
\Psi(1)=\cos \alpha|1\rangle+\sin \alpha|-1\rangle ; \quad \Psi(0)=|0\rangle
$$

и

$$
\begin{gathered}
\Psi(-1)=-\sin \alpha|1\rangle+\cos \alpha|-1\rangle, \\
\Phi\left(\frac{1}{2}\right)=\left|\frac{1}{2}\right\rangle \text { и }\left|-\frac{1}{2}\right\rangle .
\end{gathered}
$$

Связь спиновых операторов с операторами Хаббарда, построенных на базисе собственных функций гамильтониана (8) теперь имеет вид

$$
\begin{gathered}
S_{n}^{z}=\cos 2 \alpha\left(X_{n}^{11}-X_{n}^{-1-1}\right)-\sin 2 \alpha\left(X_{n}^{1-1}+X_{n}^{-11}\right) \\
S_{n}^{+}=\sqrt{2}\left[\sin \alpha\left(X_{n}^{01}-X_{n}^{-10}\right)+\cos \alpha\left(X_{n}^{0-1}+X_{n}^{10}\right)\right] \\
S_{n}^{-}=\left(S_{n}^{+}\right)^{+},
\end{gathered}
$$


где $\alpha-$ параметр унитарного $u-v$ преобразования, определяемый соотношением

$$
\bar{H}_{S} \sin 2 \alpha=-B_{2}^{2} \cos 2 \alpha .
$$

Из связи спиновых операторов с операторами Хаббарда можно определить параметры порядка первой подрешетки, как функцию $\alpha$ :

$$
\left\langle S^{z}\right\rangle=\cos 2 \alpha, \quad q_{2}^{2}=\left\langle O_{2}^{2}\right\rangle=\sin 2 \alpha, \quad q_{2}^{0}=\left\langle O_{0}^{2}\right\rangle=1 .
$$

Вторая подрешетка описывается лишь дипольным параметром порядка $\left\langle\sigma^{z}\right\rangle$ и выполняет роль „подмагничивающего“ поля.

\section{3. Анализ свободной энергии}

Поскольку мы рассматриваем систему при низких температурах, то плотность свободной энергии практически совпадает с энергетическими уровнями магнитного иона основного состояния. Как следует из соотношений (7), нижайшими уровнями первой и второй подрешеток, являются уровни $E_{1}$ и $\varepsilon_{\frac{1}{2}}$, соответственно. Следовательно, плотность свободной энергии рассматриваемого ферримагнетика можно представить в виде

$$
F=E_{1}+\varepsilon_{\frac{1}{2}}
$$

Учитывая соотношения (4) и (7), для плотности свободной энергии получим

$$
\begin{aligned}
F= & -\frac{1}{12} K_{0}-\frac{1}{4} \beta-\frac{1}{2} J_{0}^{(1)}\left\langle\sigma^{z}\right\rangle^{2}-\frac{1}{2}\left[J_{0}^{(2)}-K_{0}\right]\left\langle S^{z}\right\rangle^{2} \\
& +\frac{1}{2} A_{0}\left\langle\sigma^{z}\right\rangle\left\langle S^{z}\right\rangle+\frac{1}{12} \beta \sin 2 \alpha .
\end{aligned}
$$

Учитывая, что $\left\langle\sigma^{z}\right\rangle=1 / 2,\left\langle S^{z}\right\rangle=\cos 2 \alpha$, а также то, что константа межподрешеточного взаимодействия $A<0$, получим следующее выражение:

$$
\begin{aligned}
F= & -\frac{1}{4}\left[\beta+\frac{4}{3} K_{0}+\frac{1}{2} J_{0}^{(1)}\right]-\frac{1}{4}\left|A_{0}\right| \cos 2 \alpha \\
& +\frac{1}{12} \beta \sin 2 \alpha-\frac{1}{2}\left[J_{0}^{(2)}-K(0)\right] \cos ^{2} 2 \alpha .
\end{aligned}
$$

Анализ плотности свободной энергии (9) позволяет определить параметр $\alpha u-v$ преобразования при различных соотношениях материальных параметров системы.

В общем случае уравнение на параметр $\alpha$ в общем случае имеет вид

$$
\frac{\left|A_{0}\right|}{2} \sin 2 \alpha+\frac{\beta}{6} \cos 2 \alpha+2\left(J_{0}^{(2)}-K_{0}\right) \cos 2 \alpha \sin 2 \alpha=0 .
$$

Как следует из уравнения (10), намагниченность подрешетки с $S=1$ существенно зависит от соотношения материальных параметров, причем намагниченность подрешетки со спином $1 / 2$ остается постоянной, и играет роль
„Подмагничивающего“ поля. Необходимо отметить, что условие $\left\langle\sigma^{z}\right\rangle=1 / 2$ возникает естественным образом из связи $z$-ой компоненты оператора $\sigma$ с операторами Хаббарда $Y^{m^{\prime} m}$ и является точным в рассматриваемом нами случае $T=0$.

Рассмотрим подробнее решения уравнения (10) при различных соотношениях материальных параметров и низких температурах.

Так, если константа одноионной анизотропии много меньше билинейного и биквадратичного обменных взаимодействий, а билинейный обмен, в свою очередь, превосходит биквадратичный $\left(J_{0}>K_{0} \gg \beta\right)$, то при таком соотношении материальных параметров решение уравнения (10) можно представить в виде

$$
\sin 2 \alpha=-\frac{\beta / 3}{4\left(J_{0}^{(2)}-K_{0}\right)+\left|A_{0}\right|} .
$$

Поскольку мы предполагаем, что константа одноионной анизотропии является самым малым параметром системы, а $J_{0}>K_{0}$, то $\sin 2 \alpha \sim 0$, и, следовательно, $\cos 2 \alpha \sim 1$, т.е. намагниченность первой подрешетки практически достигает своего максимально возможного значения $\left\langle S^{z}\right\rangle \approx 1$, таким образом состояние системы близко к ферримагнитному. Это означает, что в системе реализуется ферримагнитное упорядочение (FiM) c векторами состояния подрешеток

$$
|\Psi(1)\rangle=|1\rangle \quad \text { и }\left|\Phi\left(\frac{1}{2}\right)\right\rangle=\left|\frac{1}{2}\right\rangle,
$$

и параметрами порядка

$$
\left|\left\langle\sigma^{z}\right\rangle\right|=\frac{1}{2}, \quad\left\langle S^{z}\right\rangle=\cos 2 \alpha \approx 1, \quad q_{2}^{0}=1, \quad q_{2}^{2} \approx 0 .
$$

Как видно, в этом состоянии первая и вторая подрешетки близка к насыщению, но вектора намагниченности подрешеток антиколлинеарны. Необходимо отметить, что подрешетка $S=1$ достигает насыщения асимптотически, т. е. при достаточно больших значениях константы билинейного обменного взаимодействия.

Рассмотрим теперь противоположный случай, когда преобладающим параметром первой подрешетки является биквадратичное обменное взаимодействие. В этом случае решение уравнения (10) имеет вид

$$
\cos 2 \alpha=\frac{\left|A_{0}\right|}{4\left(K_{0}-J_{0}^{(2)}\right)+\beta / 3} .
$$

Поскольку $\cos 2 \alpha$ определяет средний магнитный момент (на узле) первой подрешетки, то эта величина должна быть положительной, т. е.

$$
\frac{\left|A_{0}\right|}{4\left(K_{0}-J_{0}^{(2)}\right)+\beta / 3}>0 .
$$

Кроме того, функция $\cos 2 \alpha$ ограничена. Таким образом, при $K(0)>\beta>J(0)$ в системе реализуется состояние 
с намагниченностью первой подрешетки существенно меньше максимально возможного, а вторая подрешетка сохраняет насыщенное значение намагниченности $\left(\left|\left\langle\sigma^{z}\right\rangle\right|=1 / 2\right)$. Квадрупольные параметры порядка первой подрешетки в этом случае имеют вид

$$
q_{2}^{2}=\left\langle O_{2}^{2}\right\rangle=\sin 2 \alpha<1, \quad q_{2}^{0}=\left\langle O_{2}^{0}\right\rangle=1
$$

Таким образом, в системе реализуется фаза, в которой как векторный параметр порядка первой подрешетки $\left(\left\langle S^{z}\right\rangle\right)$, так и компоненты тензора квадрупольных моментов $\left(q_{2}^{2}\right)$ первой подрешетки принимают промежуточные значения, лежащие в интервале между нулем и единицей, а вторая подрешетка играет роль постоянного „подмагничивающего поля“. Таким образом, при больших значениях константы биквадратичного обменного взаимодействия, и немалой одноионной анизотропии, в первой подрешетке возникает эффект квантового сокращения спина [23,29,34]. Такое состояние назовем квадрупольно-ферримагнитным (QFiM).

Векторы основного состояния подрешеток в QFiM-фазе имеют вид

$$
|\Psi(1)\rangle=\cos \alpha|1\rangle+\sin \alpha|-1\rangle, \quad\left|\Phi\left(\frac{1}{2}\right)\right\rangle=\left|\frac{1}{2}\right\rangle .
$$

Вектора намагниченности первой и второй подрешеток антиколлинеарны, и, следовательно, в этой фазе, с учетом квантового сокращения спина первой подрешетки $[23,29,34]$, возможна компенсация спинов подрешеток. Из условия $\left\langle S^{z}\right\rangle=-\left\langle\sigma^{z}\right\rangle$, и учитывая, что $\left\langle\sigma^{z}\right\rangle=1 / 2$, получим

$$
\frac{\left|A_{0}\right|}{4\left(K_{0}-J_{0}^{(2)}\right)+\beta / 3}=-1 / 2 .
$$

Решение этого уравнения имеет вид

$$
|A(0)|=-2\left(K_{0}-J_{0}^{(2)}\right)-\beta / 6 .
$$

Таким образом, уравнение (12) описывает поверхность в переменных $(J, K, A, \beta)$ на которой суммарный средний спин подрешеток равен нулю $\left(\left\langle S^{z}+\sigma^{z}\right\rangle=0\right)$. Необходимо подчеркнуть, что в данном случае речь идет именно о компенсации спинов подрешеток, а не о компенсации магнитных моментов подрешеток. Дело в том, что магнитный момент связан со спиновым моментом подрешеток соотношением $\mathbf{M}=-g \mu_{B} \mathbf{S}$, где $g$-фактор Ланде ( $g$-фактор). Поскольку в рассматриваемой нами модели подрешетки являются не эквивалентными, то логично считать, что и $g$-факторы подрешеток не равны, a, следовательно, не равны и магнитные моменты подрешеток на плоскости компенсации [18]. Таким образом, хотя спиновые моменты и компенсируют друг друга при определенных нами соотношениях материальных параметров, но интегральный магнитный момент при этом может быть не равен нулю, и достигать достаточно большего значения, большего, например, чем для слабых ферромагнетиков (AFM с взаимодействием Дзялошинского-Мориа). Причем, этот результирующий магнитный момент параллелен вектору антиферромагнетизма, и динамику ферримагнетика в точке компенсации можно рассматривать как „антиферромагнитную“ $[18]$.

Уравнение (12) удобнее переписать в приведенных переменных $y=|A| / K, x=J / K, y=\beta / K$. Тогда

$$
y=2 x-2-z / 6 .
$$

Необходимо отметить, что в отсутствие межподрешеточного обменного взаимодействия $(A=0)\left\langle S^{z}\right\rangle=$ $=\cos 2 \alpha=0$, т. е. параметр $\alpha=\pi / 4$. Это означает, что при $A=0$ в первой подрешетке реализуется нематическое состояние [49-54], параметры порядка которого имеют вид

$$
\left\langle S^{z}\right\rangle=0, \quad q_{2}^{2}=\left\langle O_{2}^{2}\right\rangle=1, \quad q_{2}^{0}=\left\langle O_{2}^{0}\right\rangle=1 .
$$

При этом „подмагничивающее поле“, т.е. вторая подрешетка не оказывает никакого влияния на первую.

Из равенства плотности свободной энергии в FiM и QFiM-фазах получим поверхность фазового перехода между этими фазами

$$
\left[\left|A_{0}\right|-4\left(K_{0}-J_{0}^{(2)}\right)-\beta / 3\right]^{2}+\frac{\beta}{3}\left[4\left(K_{0}-J_{0}^{(2)}\right)+\beta / 3\right]=0
$$

или в приведенных переменных $(x, y, z)$

$$
[y-4(1-x)-z / 3]^{2}+\frac{z}{3}[4(1-x)+z / 3]=0 .
$$

Полученные результаты позволяют построить фазовую диаграмму исследуемой системы, причем, ее удобнее изобразить в приведенных переменных на плоскости $(x, y)$, при различных значения $z$, т.е. при различных значениях константы одноионной анизотропии $\beta$. Схематично эта диаграмма приведена на рисунке.

Из этой фазовой диаграммы и соотношений (13) и (14) видно, что при $z=0(\beta=0)$ полученные нами результаты в точности переходят в результаты работы [38,39], в которой исследованы фазовые состояния изотропного и обменно-анизотропного негйзенберговских ферримагнетиков. Анализ полученных в данной работе результатов свидетельствует о том, что одноионная анизотропия существенно увеличивает область существования QFiM-фазы, и сдвигает как линии фазового перехода, так и линии компенсации в область больших значений билинейного обменного взаимодействия подрешетки $S=1$. Этот результат легко понять, если обратить внимание на выражение (11), из которой следует, что даже при $J_{0}^{(2)} \sim K_{0}$ среднее значение магнитного момента подрешетки с $S=1$ будет меньше номинального при больших значениях константы одноионной анизотропии $\left(\beta>\left|A_{0}\right|\right)$.

Представляет также интерес определить тип фазового перехода QFiM-FiM-фаза. Для этого рассмотрим 


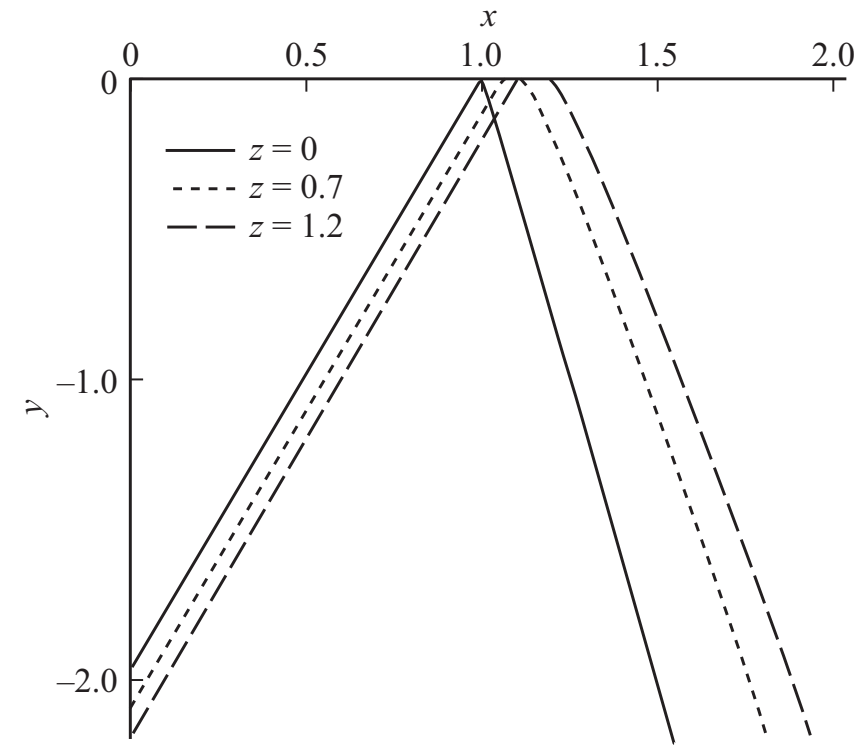

Сечение фазовой диаграммы легкоплоскостного негейзенберговского ферримагнетика при различных значениях константы одноионной анизотропии.

плотность свободной энергии (9) в окрестности фазового перехода QFiM-FiM, т.е. в окрестности линии, определяемой соотношением (14). Поскольку вторая подрешетка выполняет роль „подмагничивающего поля“, и намагниченность ее в обоих фазах одинакова и постоянна $\left(\left|\left\langle\sigma^{z}\right\rangle\right|=1 / 2\right)$, сосредоточим свое внимание на первой подрешетке. Поскольку средний магнитный момент первой подрешетки равен $\cos 2 \alpha$, то параметр $\alpha$ фактически определяет параметр порядка системы. Это утверждение требует некоторого комментария. Как уже отмечалось ранее, при анализе FiM-фазы, намагниченность подрешетки с $S=1$ достигает насыщения асимптотически. Это означает, что параметр $\alpha$ не точно равен нулю в FiM-фазе, а стремится к нулю также асимптотически при больших значениях билинейного обменного взаимодействия, и достигает точного значения $\alpha=0$ в изотропном случае при $\beta=0$. Поэтому минимум плотности свободной энергии в анизотропном случае не достигается в точке $\alpha=0$, а несколько сдвинут (в силу малости $\alpha$ ) вблизи линии фазового перехода. Раскладывая плотность свободной энергии (9) в ряд по этому параметру в QFiM-фазе в окрестности линии фазового перехода $(\alpha \rightarrow 0)$ получим

$$
F=F_{0}+A \alpha+\Lambda \alpha^{2}+B \alpha^{3}+\Theta \alpha^{4} \ldots,
$$

где

$$
\begin{gathered}
A=\frac{\beta}{6}, \quad \Lambda=2 J_{0}^{(2)}-2 K_{0}+\frac{1}{2}\left|A_{0}\right|, \quad B=-\frac{\beta}{9}, \\
\Theta=-\frac{1}{6}|A(0)|-\frac{8}{3} J_{2}(0)+\frac{8}{3} K(0),
\end{gathered}
$$

или в переменных $(x, y, z)$

$$
\begin{gathered}
A=\frac{z}{6} K_{0}, \quad B=-\frac{z}{9} K_{0}, \quad \Lambda=\frac{K(0)}{2}(4 x-4+y), \\
\Theta=\frac{K(0)}{6}(-y-16 x+16) .
\end{gathered}
$$

Наличие линейного по $\alpha$ слагаемого в выражении (15) свидетельствует о том, что подрешетка $S=1$ является ненасыщенной в FiM-фазе. Такое поведение параметра $\alpha$ связано с наличием квадрупольных средних вида $\left\langle S^{i} S^{j}+S^{j} S^{i}\right\rangle$, которое в рассматриваемом случае есть

$$
q_{2}^{2}=\left\langle\left(S^{x}\right)^{2}-\left(S^{y}\right)^{2}\right\rangle=\frac{1}{2}\left\langle\left(S^{+}\right)^{2}+\left(S^{-}\right)^{2}\right\rangle=\sin 2 \alpha .
$$

Анализ плотности свободной энергии (15) позволяет трактовать фазовый переход QFiM-FiM-фаза как фазовый переход первого рода. Поскольку коэффициент $\Theta>0$ в QFiM-фазе, то кубическая парабола, определяемая уравнением

$$
A+2 \Lambda \alpha+3 B \alpha^{2}+4 \Theta \alpha^{3}=0
$$

имеет два минимума, ни один из котрых не совпадает с точкой $\alpha=0$.

В случае изотропного ферримагнетика плотность свободной энергии в окрестности линии фазового перехода QFiM-FiM имеет

$$
F=F_{0}+\Lambda \alpha^{2}+\Theta \alpha^{4}+\ldots
$$

а величины $\Lambda$ и $\Theta$ имеют вид, приведенный выше. Следовательно, в случае изотропного негйзенберговского ферримагнетика рассматриваемый фазовый переход является переходом второго рода.

\section{4. Обсуждение результатов}

Проведенные исследования показали, что в негейзенберговском ферримагнетике с одноионной анизотропией типа ,легкая плоскость“ и подрешетками $S=1$ и $\sigma=1 / 2$ возможна реализация как ферримагнитного состояния с интегральным магнитным моментом $\left\langle S^{z}+\sigma^{z}\right\rangle=1 / 2$, так и фазы, в которой одновременно присутствуют как векторные параметры порядка первой и второй подрешеток $\left(\left\langle S^{z}\right\rangle,\left\langle\sigma^{z}\right\rangle\right)$, так и тензорный параметр порядка для первой подрешетки, наличие которого, обусловлено как влиянием биквадратичного обменного взаимодействия первой подрешетки, так и учетом в этой подрешетке легкоплоскостной анизотропии. Учет этих взаимодействий приводит к квантовому сокращению спина первой подрешетки, но не влияет на величину магнитного момента второй подрешетки. При этом, вторая подрешетка играет роль „подмагничивающего“ поля, и не позволяет ни при каких значениях биквадратичного обменного взаимодействия и одноионной анизотропии перевести первую подрешетку в состояние спинового 
нематика [31]. Эту фазу мы назвали квадрупольноферримагнитной. Поскольку в этой фазе среднее значение спина первой подрешетки изменяется в зависимости от соотношения материальных параметров подрешетки, то в этом состоянии возможна компенсация спинов подрешеток. Нами получено уравнение линии компенсации в пространстве материальных параметров, а также линии фазового перехода „ферримагнитная - квадрупольно-ферримагнитная фаза“. Необходимо отметить, что полученные нами результаты согласуются с результатам работ $[38,39]$, в которых исследовались своства изотропного и обменно-анизотропного негейзенберговских ферримагнетиков с подрешетками $S=1, \sigma=1 / 2$. Как уже отмечалось ранее, учет легкоплоскостной одноионной анизотропии в подрешетке с $S=1$ существенно расширяет область устойчивости QFiM-фазы по сравнению с зотропным ферриманетиком, и что наиболее интересно, делает переход QFiM-FiM фазовым переходом первого рода. Такое поведение рассматриваемой системы требует подробного исследования динамики системы. Также, большой интерес представляет поведение спектров элементарных возбуждений в окрестности линии компенсации. Эти исследования авторы надеются сделать в ближайшем будущем.

\section{Финансирование работы}

Работа выполнена при финансовой поддержке РФФИ и Республики Крым, проект № 20-42-910003 (О.А. Космачев, Е.А. Ярыгина, Я.Ю. Матюнина).

Исследование финансировалось РФФИ по проекту № 20-32-90027 (Е.А. Ярыгина).

\section{Конфликт интересов}

Авторы заявляют, что у них нет конфликта интересов.

\section{Список литературы}

[1] H.V. Gomonay V.M. Loktev. Low. Temp. Phys. 40, 17 (2014). https://doi.org/10.1063/1.4862467

[2] V. Baltz, A. Manchon, M. Tsoi, T. Moriyama, T. Ono, Y. Tserkovnyak, Rev. Mod. Phys. 90, 015005 (2018). https://doi.org/10.1103/RevModPhys.90.015005

[3] M.B. Jungfleisch, W. Zhang, A. Hoffmann. Phys. Lett. A 382, 865 (2018). https://doi.org/10.1016/j.physleta.2018.01.008

[4] H.V. Gomonay, V.M. Loktev. Phys. Rev. B 81, 144427 (2010). https://doi.org/10.1103/PhysRevB.81.144427

[5] O.A. Tretiakov, D. Clarke, G.-W. Chern, Y.B. Bazaliy, O. Tchernyshyov, Phys. Rev. Lett. 100, 127204 (2008). https://doi.org/10.1103/PhysRevLett.100.127204

[6] E.G. Galkina, B.A. Ivanov, S. Savel'ev, F. Nori. Phys. Rev. B 77, 134425 (2008). https://doi.org/10.1103/PhysRevB.77.134425

[7] O. Gomonay, T. Jungwirth, J. Sinova, Phys. Rev. Lett. 117, $017202(2016)$.

https://doi.org/10.1103/PhysRevLett.117.017202
[8] E.G. Galkina, B.A. Ivanov. Low Temp. Phys. 44, 618 (2018). https://doi.org/10.1063/1.5041427

[9] R. Cheng, D. Xiao, A. Brataas. Phys. Rev. Lett. 116, 207603 (2016). https://doi.org/10.1103/PhysRevLett.116.207603

[10] R. Khymyn, I. Lisenkov, V. Tyberkevych, B.A. Ivanov, A. Slavin. Sci. Rep. 7, 43705 (2017). https://doi.org/10.1038/srep43705

[11] O.R. Sulymenko, O.V. Prokopenko, V.S. Tiberkevich, A.N. Slavin, B.A. Ivanov, R. Khymyn. Phys. Rev. Appl. 8, 064007 (2017). https://doi.org/10.1103/PhysRevApplied.8.064007

[12] B.A. Ivanov, A.L. Sukstanski. JETP 84, 370 (1983).

[13] K.-J. Kim, S.K. Kim, Y. Hirata, Se-Hyeok Oh, T. Tono, D.-H. Kim, T. Okuno, W.S. Ham, S. Kim, G. Go, Y. Tserkovnyak, A. Tsukamoto, T. Moriyama, K.-J. Lee, T. Ono. Nature Mater. 16, 1187 (2017). https://doi.org/10.1038/nmat4990

[14] E.G. Galkina, C.E. Zaspel, B.A. Ivanov, N.E. Kulagin, L.M. Lerman. JETP Lett. 110, 474 (2019). https://doi.org/10.1134/S0370274X1919007X

[15] S.K. Kim, Y. Tserkovnyak. Appl. Phys. Lett. 111, 032401 (2017). https://doi.org/10.1063/1.4985577

[16] C.E. Zaspel, E.G. Galkina, B.A. Ivanov. Phys. Rev. Appl. 12, 044019 (2019). https://doi.org/10.1103/PhysRevApplied.12.044019

[17] I. Lisenkov, R. Khymyn, J. Åkerman, N.X. Sun, B.A. Ivanov. Phys. Rev. B 100, 100409(R) (2019). https://doi.org/10.1103/PhysRevB.100.100409

[18] B.A. Ivanov. Low Temp. Phys. 45, 935 (2019). https://doi.org/10.1063/1.5121265

[19] I. Radu, K. Vahaplar, C. Stamm, T. Kachel, N. Pontius, H.A. Dürr, T.A. Ostler, J. Barker, R.F.L. Evans, R.W. Chantrell, A. Tsukamoto, A. Itoh, A. Kirilyuk, Th. Rasing, A.V. Kime. Nature London 472, 205 (2011). https://doi.org/10.1038/nature09901

[20] T.A. Ostler, J. Barker, R.F.L. Evans, R. Chantrell, U. Atxitia, O. Chubykalo-Fesenko, S. El Moussaoui, L. Le Guyader, E. Mengotti, L.J. Heyderman, F. Nolting, A. Tsukamoto, A. Itoh, D.V. Afanasiev, B.A. Ivanov, A.M. Kalashnikova, K. Vahaplar, J. Mentink, A. Kirilyuk, Th. Rasing, A.V. Kimel. Nature Commun. 3, 666 (2012). https://doi.org/10.1038/ncomms1666

[21] J.H. Mentink, J. Hellsvik, D.V. Afanasiev, B.A. Ivanov, A. Kirilyuk, A.V. Kimel, O. Eriksson, M.I. Katsnelson, Th. Rasing. Phys. Rev. Lett. 108, 057202 (2012). https://doi.org/10.1103/PhysRevLett.108.057202

[22] V.G. Bar'yakhtar, V.I. Butrim, B.A. Ivanov. JETP Lett. 98, 289 (2013). https://doi.org/10.1134/S0021364013180057

[23] Yu.A. Fridman, O.A. Kosmachev. Phys. Solid State 51, 6, 1167 (2009). DOI: 10.1134/S1063783409060146

[24] E.L. Nagaev. Sov. Phys. Usp. 25, 31 (1982).

[25] V.M. Loktev, V.S. Ostrovskii. Low Temp. Phys. 20, 775 (1994).

[26] T. Moriya. Phys. Rev. 117, 635 (1960).

[27] Yu.N. Mitsay, Yu.A. Fridman, D.V. Spirin, M.S. Kochmanski. Acta Phys. Pol. 97, 355 (2000). https://doi.org/10.12693/APhysPolA.97.355

[28] Yu.A. Fridman, O.A. Kosmachev. JMMM. 236, 272 (2001). https://doi.org/10.1016/S0304-8853(01)00464-4

[29] E.G. Galkina, V.I. Butrim, Yu.A. Fridman, B.A. Ivanov, Franco Nori. Phys. Rev. B 88, 144420 (2013). https://oi.org/10.1103/PhysRevB.88.144420 
[30] E.G. Galkina, B.A. Ivanov, V.I. Butrim. Low Temp. Phys. 40, 635 (2014). https://doi.org/10.1063/1.4890989

[31] A.F. Andreev, I.A. Grishchuk. Sov. Phys. JETP 60, 267 (1984).

[32] E.L. Nagaev. Magnets with Complex Exchange Interactions. Nauka, Moscow (1988).

[33] B.A. Ivanov, A.K. Kolezhuk. Phys. Rev. B 68, 052401 (2003). https://doi.org/10.1103/PhysRevB.68.052401

[34] V.G. Bar'yakhtar, V.I. Butrim, A.K. Kolezhuk, B.A. Ivanov. Phys. Rev. B 87, 224407 (2013).

DOI: 10.1103/PhysRevB.87.224407

[35] E.G. Galkina, B.A. Ivanov, O.A. Kosmachev, Yu.A. Fridman. Low Temp. Phys. 41, 382 (2015). http://dx.doi.org/10.1063/1.4921470

[36] Yu.A. Fridman, O.A. Kosmachev, Ph.N. Klevets. JMMM 325, 125 (2013). http://dx.doi.org/10.1016/j.jmmm.2012.08.027

[37] A. Läuchli, G. Schmid, S. Trebst. Phys. Rev. B 74, 144426 (2006). https://doi.org/10.1103/PhysRevB.74.144426

[38] A.V. Krivtsova, Ya.Yu. Matyunina, E.A. Polyanskaya, O.A. Kosmachev, Yu.A. Fridman. JMMM 513, 167178 (2020). https://doi.org/10.1016/j.jmmm.2020.167178

[39] A.V. Krivtsova, Ya.Yu. Matyunina, Yu.A. Fridman. JETP 131, 302 (2020). DOI: 10.1134/S1063776120060059

[40] N. Papanikolaou. Nucl. Phys. B 305, 367 (1988).

[41] A. V. Chubukov. J. Phys. Condens. Matter 2, 1593 (1990).

[42] K. Stevens. Proc. Phys. Soc. A 65, 209 (1952).

[43] R.O. Zaitsev. Sov. Phys. JETP 41, 1, 100 (1975).

[44] V.V. Val'kov. Sov. J. Theor. Meth. Phys. 76, 766 (1988).

[45] Yu.A. Fridman, O.A. Kosmachev, Ph.N. Klevets. JMMM 320, 435 (2008). doi: 10.1016/j.jmmm.2007.07.001

[46] Yu.N. Mitsai, Yu.A. Fridman. TMF 81, 263 (1989).

Редактор Д.В. Жуманов 